\title{
Quantifying the robustness of process manufacturing concept - A medical product case study
}

\author{
Boorla, S.M. ${ }^{\mathrm{a},{ }^{*}}$, Troldtoft, M.E. ${ }^{\mathrm{b}}$, Eifler, T. ${ }^{\mathrm{a}}$, Howard, T.J. ${ }^{\mathrm{a}}$ \\ ${ }^{a}$ Technical University of Denmark, Department of Mechanical Engineering, Denmark \\ ${ }^{\mathrm{b}}$ Prolnvent A/S, Hørsholm, Denmark
}

\begin{abstract}
A B S T R A C T
Product robustness refers to the consistency of performance of all of the units produced. It is often the case that process manufactured products are not designed concurrently, so by the end of the product design phase the Process Manufacturing Concept (PMC) has yet to be decided. Allocating process capable tolerances to the product during the design phase is therefore not possible. The robustness of the concept (how capable it is to achieve the product specification), only becomes clear at this late stage and thus after testing and iteration. In this article, a method for calculating the unit-to-unit robustness of an early-stage for a PMC is proposed. The method uses variability and adjustability information from the manufacturing concept in combination with sensitivity information from products' design to predict its functional performance variation. A Technology maturation factor for addressing varied process capability confidence was applied. A four-step process of Define, Connect, Map and Quantify was proposed for calculating PMC robustness and was tested for a wound-care product. The results show that the method was applicable and enabled PMC selection based on quantified robustness. The case also demonstrates that higher robustness is possible even at higher parameter variability with suitable measurements and adjustability.
\end{abstract}

\section{ARTICLE INFO}

Keywords:

Product robustness

Process manufacturing concept

Smart process manufacturing

Variation compensation

Industry 4.0

*Corresponding author:

srimbo@mek.dtu.dk

(Boorla, S.M.)

Article history:

Received 13 February 2017

Revised 10 April 2017

Accepted 18 April 2017

\section{References}

[1] Howard, T.J., Eifler, T., Pedersen S.N., Göhler S.M., Boorla, S.M., Christensen, M.E. (2017). The variation management framework (VMF): A unifying graphical representation of robust design, Quality Engineering, 1-10, doi: 10.1080/08982112.2016.1272121.

[2] Saha, A., Ray, T. (2011). Practical robust design optimization using evolutionary algorithms, Journal of Mechanical Design, Vol. 133, No. 10, doi: 10.1115/1.4004807.

[3] Ebro, M., Howard, T.J. (2016). Robust design principles for reducing variation in functional performance, Journal of Engineering Design, Vol. 27, No. 1-3, 75-92, doi: 10.1080/09544828.2015.1103844.

[4] Ebro, M., Howard, T.J., Rasmussen, J.J. (2012). The foundation for robust design: Enabling robustness through kinematic design and design clarity, In: Proceedings of the $12^{\text {th }}$ International Design Conference, Dubrovnik, Croatia, 817-826.

[5] Göhler, S.M., Ebro, M., Howard, T.J. (2016). Mechanisms and coherences of robust design methodology: A robust design process proposal, Total Quality Management \& Business Excellence, 1-21, doi: 10.1080/14783363.2016. 1180952.

[6] Downey, K., Parkinson, A., Chase, K. (2003). An introduction to smart assemblies for robust design, Research in Engineering Design, Vol. 14, No. 4, 236-246, doi: 10.1007/s00163-003-0041-5.

[7] Helten, K., Hellenbrand, D., Lindemann, U. (2009). Product robustness as a basis for the improvement of production planning processes - Key factors in early design phases, In: DS 58-7: Proceedings of the the 17th International Conference on Engineering Design, Palo Alto, CA, USA, 197-206. 
[8] Boorla, S.M., Howard, T.J. (2016). Production monitoring system for understanding product robustness, Advances in Production Engineering \& Management, Vol. 11, No. 3, 159-172, doi: 10.14743/apem2016.3.217.

[9] Boer, H. (2016). Advanced manufacturing technologies and strategically flexible production. A review and outlook. In: Bartezzaghi, E., Cagliano, R., Caniato, F., Ronchi, S. (eds.), A Journey through Manufacturing and Supply Chain Strategy Research: A Tribute to Professor Gianluca Spina, Springer, Switzerland, 71-110, doi: 10.1007/9783-319-31104-3 4.

[10] Davis, J.F., Edgar, T.F. (2009). Smart process manufacturing: An operations and technology roadmap, from https://smartmanufacturingcoalition.org/sites/default/files/spm - an operations and technology roadmap.pdf accessed September 9, 2016.

[11] Yu, L.X. (2008), Pharmaceutical quality by design: Product and process development, understanding, and control, Pharmaceutical research, Vol. 25, No. 4, 781-791, doi: 10.1007/s11095-007-9511-1.

[12] Ierapetritou, M.G., Ramachandran, R. (eds.), (2016), Process Simulation and Data Modeling in Solid Oral Drug Development and Manufacture, Springer, New York, USA, doi: 10.1007/978-1-4939-2996-2.

[13] Davis, J.F., Edgar, T.F. (2008). Smart process manufacturing - A vision of the future, In: Proceedings of AIChE100 2008 AIChE Annual Meeting, Philadelphia, USA.

[14] Rathinasabapathy, R., Elsass, M.J., Josephson, J.R., Davis, J.F. (2016). A smart manufacturing methodology for real time chemical process diagnosis using causal link assessment, American Institute of Chemical Engineers Journal, Vol. 62, No. 9, 3420-3431, doi: 10.1002/aic.15403.

[15] Brodsky, A., Shao, G., Riddick, F.J. (2016). Process analytics formalism for decision guidance in sustainable manufacturing, Journal of Intelligent Manufacturing, Vol. 27, No. 3, 561-580, doi: 10.1007/s10845-014-0892-9.

[16] Mankins, J.C. (2009). Technology readiness assessments: A retrospective, Acta Astronautica, Vol. 65, No. 9-10, 1216-1223, doi: 10.1016/j.actaastro.2009.03.058.

[17] Pedersen, S.N., Christensen, M.E., Howard, T.J. (2016). Robust design requirements specification: A quantitative method for requirements development using quality loss functions, Journal of Engineering Design, Vol. 27, No. 8, 544-567, doi: 10.1080/09544828.2016.1183163.

[18] Franceschini, F., Rupil, A. (1999). Rating scales and prioritization in QFD, International Journal of Quality \& Reliability Management, Vol. 16, No. 1, 85-97, doi: 10.1108/02656719910250881.

[19] Chakhunashvili, A., Barone, S., Johansson, P., Bergman, B. (2009). Robust product development using variation mode and effect analysis, In: Bergman, B., de Mare, J., Loren, S., Svensson, T. (eds.), Robust Design Methodology for Reliability: Exploring the Effects of Variation and Uncertainty, Wiley, New York, USA, 57-70, doi: 10.1002/9780470748794.ch5.

[20] Saldivar, A.A.F., Goh, C., Chen, W.-N., Li, Y. (2016). Self-organizing tool for smart design with predictive customer needs and wants to realize Industry 4.0. In: Proceedings of 2016 IEEE Congress on Evolutionary Computation, Vancouver, Canada, doi: 10.1109/CEC.2016.7748366.

[21] Lee, J., Bagheri, B., Kao, H.-A. (2015). A cyber-physical systems architecture for industry 4.0-based manufacturing systems, Manufacturing Letters, Vol. 3, 18-23, doi: 10.1016/j.mfglet.2014.12.001. 
APEM
Advances in Production Engineering \& Management

Letnik 12 | Številka 2 | Junij 2017 | Strani 127-138

https://doi.org/10.14743/apem2017.2.245
ISSN 1854-6250

Spletna stran: apem-journal.org

Izvirni znanstveni članek

\title{
Ovrednotenje robustnosti zasnove proizvodnje s procesnim pristopom - študija primera medicinskih izdelkov
}

\author{
Boorla, S.M..$^{\mathrm{a},{ }^{*}}$, Troldtoft, M.E. ${ }^{\mathrm{b}}$, Eifler, T. ${ }^{\mathrm{a}}$, Howard, T.J. ${ }^{\mathrm{a}}$ \\ ${ }^{a}$ Technical University of Denmark, Department of Mechanical Engineering, Denmark \\ ${ }^{\mathrm{b}}$ Prolnvent A/S, Hørsholm, Denmark
}

\begin{abstract}
POVZETEK
Robustnost izdelka je definirana kot zanesljivost delovanja vseh proizvedenih enot. Pogosto se zgodi, da izdelki, izdelani s procesnim pristopom, niso načrtovani hkrati s proizvodnim procesom, zato je do zaključka oblikovanja izdelka treba večkrat prilagoditi proizvodni proces. Zato tudi določitev procesno pogojenih odstopanj med izdelki v fazi zasnove ni mogoča. Robustnost proizvodnega procesa (kako sposoben je ta doseči specifikacijo izdelka), postane jasna šele $\mathrm{v}$ poznejših fazah ali po testiranju in ponovitvah. $\mathrm{V}$ tem članku je predlagana metoda za izračun robustnosti tipa izdelek-do-izdelek v zgodnji fazi zasnove proizvodnje s procesnim pristopom. Metoda uporablja podatke $o$ spremenljivosti in prilagodljivosti zasnove proizvodnega procesa $\mathrm{v}$ kombinaciji s podatki o občutljivosti iz faze zasnove izdelka, da predvidi variabilnost $\mathrm{v}$ uporabnosti izdelkov. Uporabljen je faktor zrelosti tehnologije za določitev stopnje zaupanja $\mathrm{v}$ proces. Za izračun robustnosti zasnove proizvodnje $\mathrm{s}$ procesnim pristopom je predlagan štiristopenjski postopek Definiraj, Poveži, Preslikaj in Ovrednoti. Postopek je preizkušen na izdelku za oskrbo ran. Rezultati kažejo, da je metoda uporabna in uspešna. Študija primera je pokazala, da je z ustreznimi meritvami in prilagodljivostjo, večja robustnost dosegljiva tudi pri večji variabilnosti parametrov.
\end{abstract}

\section{PODATKI O ČLANKU}

Ključne besede:

Robustnost izdelka

Zasnova proizvodnje s procesnim pristopom

Inteligentna proizvodnja s proces-

no tehniko

Kompenzacija variacij

Industrija 4.0

* Kontaktna oseba: srimbo@mek.dtu.dk (Boorla, S.M.)

Zgodovina članka:

Prejet 13. februarja 2017

Popravljen 10. aprila 2017

Sprejet 18. aprila 2017 